\title{
Hydrodynamics of surface irrigation: vertical structure of the surge front
}

Received: 10 June 1993

\begin{abstract}
A model for surface irrigation is developed that allows the determination of the vertical structure of the velocity profile in the vicinity of the wave front. The pressure is not assumed to be hydrostatically distributed and no assumptions are made regarding the shape of the freesurface profile. The turbulent kinetic energy and rate of dissipation are computed by a two-equation model and accurate determination of the bottom shear makes possible the analysis of particle suspension. The model is based on a two-dimensional finite element model in the vertical plane and uses the kinematic condition for determining the position of the free surface. It also incorporates a numerical technique for describing surface penetration and wave breaking by combining a Lagrangian approach that allows the computational nodes to move individually and then automatically reshapes the element grid. The potential value of the model lies in its ability to provide information on vertical mixing, settling and suspension of contaminated solids commonly found in irrigation applications.
\end{abstract}

Successful models of surface irrigation hydraulics have been developed by physical approximations based on the postulation that small influences in the description of freesurface flows can be neglected. Three-dimensional problems have been re-formulated by averaging in directions transverse to the main propagation direction, leading to one-dimensional models of overland flow. Energy dissipation is presumed to originate solely from the interface between the fluid and the channel bed, surface tension is ignored and gravitation is the only body force considered. Infiltration is treated as a volumetric abstraction with only small dynamic effects on the flow. In particular, the influence of bed suction on the shape of the surge front is ignored although it is understood that infiltration rates achieve

N. D. Katopodes $(\bowtie)$

Department of Civil and Environmental Engineering,

Univeristy of Michigan,

Ann Arbor, MI 48109, USA their maximum values in the vicinity of the wave front. The mathematical implementation of this approach, commonly known as shallow-water theory, has yielded models for border and furrow flow that duplicate accurately laboratory and field measurements (Sakkas and Strelkoff 1974, Katopodes and Strelkoff 1977). In most cases, the inertia of the flow is also negligible, allowing further simplification of the model (Strelkoff and Katopodes 1977).

A few additional assumptions need to be made for the dynamics of the region near the wave front, where the flow depth becomes very small and finally goes to zero at the tip of the wave. The approximate expressions for bed resistance and seepage become singular if the depth vanishes, resulting in infinite intensities for bed shear and infiltration. The wave front is commonly treated as a region of uniform flow (Whitham 1954, Tinney and Bassett 1961), with its propagation speed determined by global conservation of volume and momentum or equilibrium forces in the case of noninertial flow. The predictive ability of the shallow-water and zero-inertia models has been confirmed in numerous studies and there is little doubt that these models describe correctly the physics of the problem. Recently, however, increased attention has been focused on the transport of chemicals by surface irrigation as opposed to the optimum distribution of soil moisture, which has been the typical goal of modeling over the last twenty years. The transport problem consists of two phases, first, the introduction of fertilizers and pesticides in irrigation water during what is now known as chemigation, and second, the uptake of these chemicals from the soil's surface by the irrigation wave. Both phases represent overland flow problems coupled with subsurface flow, but more importantly, require knowledge of the vertical structure of the flow in the surface domain. For suspension of particulate matter, dissolution and turbulent mixing are mechanisms that are not present in the shallow-water theory.

The removal of the fundamental hypothesis of shallowwater theory requires that the problem be solved in at least two space dimensions, namely the vertical plane. The resulting problem is difficult to formulate, is computationally intensive, and requires a new set of assumptions to be 
made regarding its internal structure and certain singularities that arise in the new formulation (Huh and Scriven 1971, Kistler and Scriven 1983). A model that computes the location of the free surface, on the other hand, is not subject to any of the limitations of vertically integrated equations. The numerical computation can be made increasingly accurate near the tip of the wave front by gradual grid refinement. Bed resistance and infiltration can be represented by processes and parameters that are related to the actual roughness characteristics of the bed and permeability of the soil, as opposed to the semi-empirical parameters commonly used by vertically averaged models.

This paper describes the development of a numerical model for the simulation of free-surface flow on an initially dry, porous bed. This is accomplished by numerical solution of the Navier-Stokes equations by means of the finiteelement method. For large scale applications, the two-dimensional computation is restricted to the region of the wave front since a short distance upstream a fully developed vertical structure is encountered. The model simply converts automatically to shallow-water or zero-inertia theory beyond that section. This results in significant reduction in computational effort without loss of important information.

For turbulent flow, a widely accepted two-equation closure model is employed together with certain approximations near the bed and free-surface boundaries. The turbulence model has a limited accuracy, but the requirements of the flow problem considered in this work are well within the model's applicability range. At high Reynolds numbers, an upwind formulation known as the Petrov-Galerkin method of weighted residuals is introduced for the suppression of nonlinear instabilities. The main difficulty encountered in the simulation concerns the propagation of the wave front and the associated deformation of the computational grid. Two different techniques are presented for the resolution of this difficulty, and their relative advantages are examined.

Bed permeability resulting in seepage through the channel bed needs to be computed by simultaneous solution of the associated unsaturated flow problem in the sub-surface domain. Although this is necessary for the complete formulation of the flow and transport problem of contaminants in irrigation water, in this paper bed seepage is introduced as a boundary condition. The rate of infiltration is considered independent of the surface depth and velocity and is computed by means of Kostiakov's empirical equation. This results in clarity of the surace model presentation and significant reduction of computational effort, without limiting the generality of the results of the surge front model.

\section{Governing equations}

The equations that describe the motion of a water wave propagating on a dry, porous bed in the vertical plane cor- respond to the laws of conservation of mass and momentum applied to the longitudinal $x_{1}$ and vertical $x_{2}$ directions. These are known as the Navier-Stokes equations of motion and the continuity equation that can be written as follows (Schlichting 1979)

$$
\begin{aligned}
& \frac{\partial u_{i}}{\partial x_{i}}=0 \quad i=1,2 \\
& \rho\left(\frac{\partial u_{i}}{\partial t}+u_{j} \frac{\partial u_{i}}{\partial x_{j}}\right)=-\frac{\partial p}{\partial x_{i}}+\frac{\partial}{\partial x_{j}}\left[\mu\left(\frac{\partial u_{i}}{\partial x_{j}}+\frac{\partial u_{j}}{\partial x_{i}}\right)\right]+\rho g_{i} \\
& i=1,2 ; \quad j=1,2
\end{aligned}
$$

where $u_{i}$ are the velocity components in the $x_{1}$ and $x_{2} \mathrm{di}$ rections, respectively; $\rho$ is the water mass density, $p$ is the pressure intensity and $g_{i}$ are the components of the gravitational vector. In Eqs. (1) and (2) the index summation notation is implied, i.e., a repeated index within the same term implies summation over all possible values of the index. Assuming that the density is constant, the variables $u_{i}$ and $p$ can be decomposed in terms of a mean and a fluctuating quantity (Rodi 1988), as follows:

$u_{i}=U_{i}+u_{i}^{\prime} \quad p=P+p^{\prime}$

with

$$
\begin{gathered}
U_{i}=\frac{1}{T} \int_{t_{0}}^{t_{0}+T} u_{i} \mathrm{~d} t \\
P=\frac{1}{T} \int_{t_{0}}^{t_{0}+T} p \mathrm{~d} t
\end{gathered}
$$

where $U_{i}, P$ are time-averaged quantities, and $u_{i}^{\prime}, p^{\prime}$ are fluctuating velocity components and pressure, respectively. The averaging time $T$ is considered large compared to the time scale of the turbulent fluctuations, but significantly smaller than the temporal variation of the mean flow.

Substitution of Eq. (3) in Eqs. (1) and (2) and averaging over a time period $T$, leads to the following expressions for continuity and momentum of the mean flow

$$
\begin{aligned}
& \frac{\partial U_{i}}{\partial x_{i}}=0 \\
& \rho\left(\frac{\partial U_{i}}{\partial t}+U_{j} \frac{\partial U_{i}}{\partial x_{i}}\right) \\
& \quad=-\frac{\partial P}{\partial x_{i}}+\frac{\partial}{\partial x_{j}}\left[\mu\left(\frac{\partial U_{i}}{\partial x_{j}}+\frac{\partial U_{j}}{\partial x_{i}}\right)-\rho \overline{u_{i}^{\prime} u_{j}^{\prime}}\right]+\rho g_{i}
\end{aligned}
$$

where

$$
\overline{u_{i}^{\prime} u_{j}^{\prime}}=\frac{1}{T} \int_{t_{0}}^{t_{0}+T} u_{i}^{\prime} u_{j}^{\prime} \mathrm{d} t .
$$

The stress terms $\left(\rho \overline{u_{i}^{\prime} u_{j}^{\prime}}\right)$, appearing in the momentum equation are known as the Reynolds stresses. In general, additional equations are necessary for the solution of the system of Eqs. (6) and (7). An approximate closure model 
can be formulated by means of two additional equations that describe the fate and transport of the turbulence kinetic energy $k$ and rate of dissipation $\varepsilon$ defined by

$$
k=\frac{\overline{u_{i}^{\prime} u_{j}^{\prime}}}{2} \quad \varepsilon=v \overline{\frac{\partial u_{i}^{\prime}}{\partial x_{j}} \frac{\partial u_{i}^{\prime}}{\partial x_{j}}} .
$$

The turbulence stresses can thus be expressed in a manner analogous to viscous stresses, as follows

$$
\overline{u_{i}^{\prime} u_{j}^{\prime}}=\frac{2}{3} \delta_{i j} k-v_{t}\left(\frac{\partial U_{i}}{\partial x_{j}}+\frac{\partial U_{j}}{\partial x_{i}}\right)
$$

where $\delta_{i j}$ is the kronecker delta and $v_{t}$ is the kinematic eddy viscosity defined by

$$
v_{t}=C_{\mu} \frac{k^{2}}{\varepsilon}
$$

where $C_{\mu}$ is an empirical coefficient.

The well-known $k-\varepsilon$ model (Rodi 1988) is obtained by writing transport equations for $k$ and $\varepsilon$, as follows

$$
\begin{aligned}
& \frac{\partial k}{\partial t}+U_{j} \frac{\partial k}{\partial x_{j}}=D-\varepsilon+\frac{\partial}{\partial x_{j}}\left(\frac{v_{t}}{\sigma_{k}} \frac{\partial k}{\partial x_{j}}\right) \\
& \frac{\partial \varepsilon}{\partial t}+U_{j} \frac{\partial \varepsilon}{\partial x_{j}}=\frac{\partial}{\partial x_{j}}\left(\frac{v_{t}}{\sigma_{\varepsilon}} \frac{\partial \varepsilon}{\partial x_{j}}\right)+C_{1 \varepsilon} \frac{\varepsilon}{k} D-C_{2 \varepsilon} \frac{\varepsilon^{2}}{k}
\end{aligned}
$$

where

$$
D=v_{t}\left(\frac{\partial U_{i}}{\partial x_{j}}+\frac{\partial U_{j}}{\partial x_{i}}\right) \frac{\partial U_{i}}{\partial x_{j}}
$$

$C_{1 \varepsilon}, C_{2 \varepsilon}, \sigma_{k}, \sigma_{\varepsilon}$ are also empirical constants. The commonly recommended values (Rodi 1988) are as follows: $C_{\mu}=0.09, C_{1 \varepsilon}=1.44, C_{2 \varepsilon}=1.92, \sigma_{k}=1.0$, and $\sigma_{\varepsilon}=1.3$.

Although in principle the $k-\varepsilon$ model is straightforward, difficulties are encountered in practical applications (Chapman and Kuo 1985). In particular, in the course of the iterative solution, $k$ and $\varepsilon$ occasionally are assigned negative values, which is unacceptable. There is no clear explanation of the source of this problem and, in this work, both $k$ and $\varepsilon$ are considered as strictly positive functions and are not allowed to become negative. Positivity is also enforced on the source term $D-\varepsilon$ in Eq. (12). This prevents unrealistic values of the dependent variables and improves the convergence of the iterations. In addition, the empirical modification of Gibson and Rodi (1989) adding a proximity weight to the free surface as well as the bottom is used. This was found to have beneficial effects on convergence, especially at the very shallow depths near the wave front.

\section{Boundary conditions}

A set of initial and boundary conditions that is compatible with the general equations is required for a unique solution of the system of Eqs. (6) and (7) and (12) and (13) to exist. Any flow field that satisfies continuity can be used as the initial condition, so for convenience an arbitrary wave is fabricated at the upstream end of the border, based on conservation of volume alone. It is shown that the effects of this arbitrary initial condition disappear after a few time steps, so no further consideration is given to the structure of the initial condition. Boundary conditions are needed at the channel bottom, inflow and outflow sections and at the free surface.

Ordinarily, for viscous flow near solid boundaries the no-slip condition is imposed, but this requires very accurate description of the laminar sub-layer and the region of logarithmic growth of the velocity profile near the boundary. Instead, a slip velocity boundary condition can be used by specifying a semi-empirical expression for the velocity distribution near the porous channel bed (Schlichting 1979, p. 647)

$\frac{U_{1}}{u_{*}}=\frac{1}{\kappa} \ln \frac{x_{2}}{x_{2}^{\prime}}+c+\frac{i_{f}}{4 u_{*}}\left(\frac{1}{\kappa} \ln \frac{x_{2}}{x_{2}^{\prime}}+c\right)^{2}$

where $U_{1}=$ fluid velocity parallel to the channel bed, $u_{*}=$ shear velocity at the bottom, $\kappa=$ von Karman's universal constant, $i_{f}=$ the infiltration rate, $x_{2}=$ vertical distance from the bottom, $x_{2}^{\prime}=$ distance from the bottom at which the logarithmic velocity distribution sets the velocity equal to zero, which for rough boundaries is equal to $0.033 k_{s}$, where $k_{s}=$ the equivalent roughness height, and $c=$ adjustable constant whose value is related to bottom slope and roughness.

The vertical velocity component is simply identified with the infiltration rate, i.e.,

$U_{2}=i_{f}$.

Furthermore, the following wall boundary conditions for $k$ and $\varepsilon$ are obtained by assuming that the flow is under local equilibrium near the bottom region (Alfrink and van Rijn 1983)

$$
\begin{aligned}
& k=\frac{u_{*}^{2}}{\left(C_{\mu}\right)^{0.5}} \\
& \varepsilon=\frac{u_{*}^{2}\left|u_{*}\right|}{k \delta}
\end{aligned}
$$

where $\delta$ is the thickness of the inertial sublayer, set arbitrarily equal to one half of the vertical dimension of the computational element nearest to the bottom. Numerical tests indicate that the value of $\delta$ has only a small influence on the velocity distribution in the vertical. For a given element size, best results are obtained by the aforementioned choice, attributed to the presence of the mid-side nodes used for quadratic interpolation. The overall solution improves as more elements are used to resolve the vertical profile although this also increases the computational effort required. The present model for surface irrigation requires also specification of the dependent variables at the upstream end of the field. Initially, a hypothetical inflow section is created to facilitate the application of boundary conditions. It is assumed that a segment of fully developed uniform flow exists, in which the position of the free sur- 
face is known and no infiltration takes place. The flow is then allowed to develop spatially in this region before it enters the real channel. At the inflow section, the mean flow components are given by (Alfrink and van Rijn 1983)

$$
U_{1}=\frac{u_{*}}{\kappa} \ln \frac{x_{2}}{x_{2}^{\prime}}
$$

$U_{2}=0$

$k=\frac{u_{*}}{C_{\mu}^{0.5}}\left(1-\frac{x_{2}}{H}\right)$

$\varepsilon=\frac{\left|u_{*}\right| u_{*}^{2}}{k \delta}\left(1-\frac{x_{2}}{H}\right)$

where $H=$ the total depth of flow at the inflow section. The upstream boundary condition also serves as the link with the fully developed flow away from the front at later stages of the computation. As mentioned earlier, that part of the flow is computed by a standard shallow-water model that accepts a downstream depth specification supplied by the Navier-Stokes model. $H$ is now the depth at the section where the two models are joined and the inflow rate for the front region is computed by the shallow-water model at its last computational node. In this fashion, the extent of the two-dimensional model remains approximately constant, as fully developed flow sections at its upstream end are removed and new ones are added at the front, as the wave propagates down the field.

Once the stream has reached the end of the field, downstream boundary conditions are specified either as a solid boundary or as free outflow, corresponding to ponding or runoff, respectively. The latter is easily accomplished by assuming uniform efflux conditions and setting all spatial derivatives to zero. This is particularly easy in the finite element implementation, since traction terms appear in the boundary integrals resulting from application of Green's theorem. Thus, elimination of these integrals automatically implies a traction-free outflow.

When a free surface is present, two more conditions need to be applied on the associated portion of the boundary. First, assuming that the atmosphere exerts no shear and has no inertia, equality of forces on the two sides of the interface leads to

$$
n_{j} T_{i j}-\sigma \frac{1}{R} n_{i}=0
$$

where $R$ represents the radius of curvature of the free surface, $\sigma$ is the surface tension, $n_{j}$ is the unit normal to the surface and the stress tensor $T_{i j}$ is obtained by

$$
T_{i j}=-\delta_{i j} P+\mu\left(\frac{\partial U_{i}}{\partial x_{j}}+\frac{\partial U_{j}}{\partial x_{i}}\right)-\rho \overline{u_{i}^{\prime} u_{j}^{\prime}} .
$$

In addition, since a fluid particle on the surface will remain there, and provided that there is no mass transfer through the air-water interface, the following kinematic condition is obtained

$$
\frac{\partial h}{\partial t}+U_{1} \frac{\partial h}{\partial x_{1}}=\left.U_{2}\right|_{x_{2}=h}
$$

where $h\left(x_{1}, t\right)$ is the free surface elevation from the channel bottom. This condition is limited to free surface configurations in which fluid particle penetration from within the fluid is not possible. In addition, breaking of the free surface by steepening is not allowed. For these cases different formulations are needed, as shown in a later section. In the present model, Eq. (25) is used as an additional equation and is solved simultaneously with Eqs. (6) and (7) and (12) and (13), while the dynamic surface condition, Eq. (23), is enforced as a boundary condition. The additional unknown, $h$, is then used to locate the height of the free surface above the bottom, so by an iterative process the correct location of the boundary at which the normal force vanishes is determined.

\section{Numerical solution}

The numerical solution of the governing equations is obtained in two separate steps corresponding to the mean and turbulent flow quantities. The turbulent transport equations are relatively simple to solve once the mean flow field is available. The kinetic energy and dissipation rate of turbulence may be treated similarly to matter in solution being advected, diffused, generated and destroyed. The mean flow is affected by the local values of $k$ and $\varepsilon$, but the coupling process may be implemented sequentially rather than simultaneously (Schamber 1979). During each time step, the equations of continuity, momentum and the kinematic condition at the free surface are solved simultaneously. This yields a tentative flow field and position of the free surface. Next, the turbulent transport equations are solved to obtain the distribution of $k$ and $\varepsilon$. The mean flow calculations are then repeated until the iteration converges. The main difficulty lies in the mean flow calculation due to the unknown location of the free surface and its strong nonlinear coupling with the mean flow. The use of two separate iterative process with results transferring from the one to the other appears initially to be less efficient than a single typical gradient method like Newton-Raphson. In reality, however, the difference in the scale of the variables involved makes the separation of the processes preferable. Further improvement is obtained by solving for the square roots of $k$ and $\varepsilon$ instead of their actual values (Finnie and Jeppson 1991).

The equations of momentum and continuity, together with the turbulence transport model, i.e., Eqs. (12) and (13), and the kinematic free-surface condition, Eq. (25) are discretized on a deforming finite element grid. A nine-node element is used for the approximation of the velocity components and turbulence kinetic energy and dissipation rate. This is coupled with a four node element for the pressure computation. Mixed interpolation is needed to suppress spurious modes of the numerical solution (Hood and Taylor 1974). A three node, one dimensional element is used to interpolate the free-surface elevation $h$ appearing in the kinematic free surface condition. Thus, over a single element the variables $U_{i}, P, h, k$ and $\varepsilon$ are approximated as 
follows

$U_{i} \simeq \hat{U}_{i}=\left.N_{j} U_{i j}\right|_{j=1,9}$

$P \simeq \hat{P}=\left.M_{j} P_{j}\right|_{j=1,4}$

$h \simeq \hat{h}=\left.N_{j}^{\mathrm{fs}} h_{j}\right|_{j=1,3}$

$k \simeq \hat{k}=N_{j} k_{j} I_{i=1,9}$

$\varepsilon \simeq \hat{\varepsilon}=\left.N_{j} \varepsilon_{j}\right|_{j=1,9}$

where $N_{j}$ are biquadratic basis functions, $M_{j}$ are bilinear basis functions and $N_{j}^{\mathrm{fs}}$ are one-dimensional, quadratic basis functions. $U_{i j}, P_{j}, h_{j}, k_{j}$ and $\varepsilon_{j}$ are the discrete nodal values in the approximate representation and $\hat{U}_{i}, \hat{P}, \hat{h}, \hat{k}$ and $\varepsilon$ are the element-based, approximate values of the continuous variables $U_{i}, P, h, k$ and $\varepsilon$.

Substitution of these approximate values in Eqs. (6) and (7), (12) and (13) and (25) results in residual quantities, which at the element level are defined by $\mathbf{R}^{\mathrm{e}, \mathrm{M}}, \mathbf{R}^{\mathrm{e}, \mathrm{C}}, \mathbf{R}^{\mathrm{e}, \mathrm{fs}}$, $\mathbf{R}^{\mathrm{e}, \mathrm{k}}$ and $\mathbf{R}^{\mathrm{e}, \varepsilon}$. These represent the residuals of the momentum, continuity, free-surface, turbulent kinetic energy and dissipation rate equations at the element-level, respectively.

Since the interpolated variables are an approximation to the true solution, the residuals $\mathbf{R}^{\mathrm{e}}$ are generally different than zero. Galerkin's method forces these errors to become minimum in some average sense over the element by adopting weighting functions over the element that are identical to the basis functions used for interpolation. For high values of the Reynolds number, a modified weighting function is needed because of the dominant advective terms in the transport equations for mean flow momentum and turbulent quantities. The method of Hughes and Brooks (1982) is used without modification in this work. The weighting functions appearing in the following equations are assumed to be generalized forms of the interpolation functions, so the residual equations are written for simplicity in the standard Galerkin form. It is implied that the basis functions include a perturbation form that induces upwinding when the advective component of the flow becomes dominant. This is quite simple in incompressible flow, as is the present case, and no modification of the element stiffness matrix is required. Then the weak form of the governing equations reads (Leone and Gresho 1979)

$$
\begin{aligned}
\mathbf{F}^{\mathrm{e}, \mathrm{M}}= & \int_{\Omega}\left[N_{i} \rho\left(\frac{\partial U_{i}}{\partial t}+U_{j} \frac{\partial U_{i}}{\partial x_{j}}-g_{i}\right)+\frac{\partial N_{i}}{\partial x_{j}} \hat{T}_{i j}\right] \mathrm{d} \Omega \\
& -\int_{\partial \Omega} N_{i} \hat{T}_{i j} \frac{\partial x_{j}}{\partial n} \mathrm{~d} s \\
\mathbf{F}^{\mathrm{e}, \mathrm{C}}= & -\int_{\Omega} M_{i} \frac{\partial \hat{U}_{i}}{\partial x_{i}} \mathrm{~d} \Omega \\
\mathbf{F}^{\mathrm{e}, \mathrm{fs}}= & \int_{\partial \Omega} N_{i}^{\mathrm{fs}}\left(\frac{\partial h}{\partial t}+U_{1} \frac{\partial h}{\partial h}-\left.U_{2}\right|_{X_{2}=h}\right) \mathrm{d} s
\end{aligned}
$$

$$
\begin{aligned}
\mathbf{F}^{\mathrm{e}, \mathrm{k}}=\int_{\Omega} & {\left[N_{i}\left(\frac{\partial k}{\partial t}+U_{j} \frac{\partial k}{\partial x_{j}}-D+\varepsilon\right)+\frac{\partial N_{i}}{\partial x_{j}}\left(\frac{v_{t}}{\sigma_{k}} \frac{\partial k}{\partial x_{j}}\right)\right] \mathrm{d} \Omega } \\
& -\int_{\partial \Omega} N_{i}\left(\frac{v_{t}}{\sigma_{k}} \frac{\partial k}{\partial x_{j}} \frac{\partial x_{j}}{\partial n}\right) \mathrm{d} s \\
\mathbf{F}^{\mathbf{e}, \varepsilon}= & \int_{\Omega}\left[N_{i}\left(\frac{\partial \varepsilon}{\partial t}+U_{j} \frac{\partial \varepsilon}{\partial x_{j}}-C_{1 \varepsilon} \frac{\varepsilon}{k} D+C_{2 \varepsilon} \frac{\varepsilon^{2}}{k}\right)\right. \\
& \left.+\frac{\partial N_{i}}{\partial x_{j}}\left(\frac{v_{t}}{\sigma_{\varepsilon}} \frac{\partial k}{\partial x_{j}}\right)\right] \mathrm{d} \Omega-\int_{\partial \Omega} N_{i}\left(\frac{v_{t}}{\sigma_{\varepsilon}} \frac{\partial \varepsilon}{\partial x_{j}} \frac{\partial x_{j}}{\partial n}\right) \mathrm{d} s
\end{aligned}
$$

The quantities $\mathbf{F}^{\mathrm{e}, \mathrm{M}}, \mathbf{F}^{\mathrm{e}, \mathrm{C}}, \mathbf{F}^{\mathrm{e}, \mathrm{fs}}, \mathbf{F}^{\mathrm{e}, \mathbf{k}}$ and $\mathbf{F}^{\mathrm{e}, \varepsilon}$ are approximation errors to be minimized, $\partial \Omega$ indicates the boundary interfaces of the element $\Omega, \mathbf{n}$ is the unit outward vector normal to the boundary $\partial \Omega$ and $\mathrm{d} s$ is an infinitesimal segment on the boundary curve. On the bottom and inflow boundaries, Dirichlet boundary conditions are specified, therefore the boundary integrals are dropped. On the free surface, Neumann conditions are imposed, but all associated normal fluxes are identically zero, so the boundary integrals in Eqs. (31), (34) and (35) vanish in this case as well. This implies that the dynamic boundary condition, Eq. (23), is automatically enforced and that the normal derivatives of $k$ and $\varepsilon$ are zero at the free surface.

The free surface changes its position over the course of time as a result of the propagation of the wave front on the initially dry border and dynamic adjustment of the depth of flow. As a consequence, the nodes in the computational domain need to move for the computational grid to maintain its integrity and computational effectiveness. The only nodes that move directly as a result of dynamic changes in the flow equations are those on the free surface. The rest of the nodes are then relocated suitably with the objective to minimize element distortion. Therefore, the nodal velocities are not physically related to the fluid velocity, and need to be independently computed by a simple difference scheme based on the distance traveled over a time step. In addition, a modification must be made to the time derivatives to account for node translation (Lynch and Gray 1980; Ramaswamy and Kawahara 1987). Since the nodes are moving, the basis functions $N_{i}$, which were polynomials of the independent space variables $\mathbf{x}$ only, now become implicit functions of time, as the following relation indicates

$N_{i} \equiv N_{i}(\mathbf{x}, \mathbf{X}(t))=N_{i}(\mathbf{x}, t)$

where $\mathbf{X}(t)$ are the global moving coordinates of the nodes which depend explicitly on time.

The approximate vector of time dependent variables $\mathbf{U}^{i}$, $\mathbf{P}^{i}, \mathbf{h}^{i}, \mathbf{k}^{i}$ and $\varepsilon^{i}$ can be expressed

$\mathbf{u}=\left(\mathbf{U}^{i}, \mathbf{P}^{i}, \mathbf{h}^{i}, \mathbf{k}^{i}, \varepsilon^{i}\right)^{T}$.

Since the basis functions are now time-dependent as well, the local acceleration terms read 


$$
\frac{\partial \hat{\mathbf{u}}}{\partial t}=\frac{\mathrm{d} \mathbf{u}^{i}}{\mathrm{~d} t} N^{i}+\mathbf{u}^{i} \frac{\partial N^{i}}{\partial t} .
$$

The first term on the right hand side of Eq. (38) is the time derivative of the nodal value of $\mathbf{u}$, with the partial derivative of $\mathbf{u}^{i}$ with respect to time replaced by an ordinary derivative, since $\mathbf{u}$ is a function of time only. The second term is due to the deforming boundary and the implied dependence of $N$ on time.

Equation (38) indicates that the partial derivatives of the basis functions with respect to time must be evaluated throughout the domain. This can be avoided if the time derivatives are expressed in terms of the fixed isoparametric coordinates (Lynch and Gray 1980). In this case, the local elemental coordinates do not deform with time and the basis functions at any point $\left(\xi_{0}, \eta_{0}\right)$ will not be a function of time either. Equation (38) can then be written as follows

$$
\frac{\partial \hat{\mathbf{u}}}{\partial t}=\frac{\mathrm{d} \hat{\mathbf{u}}^{i}}{\mathrm{~d} t} N^{i}(\xi, \eta)-\frac{\mathrm{d} \hat{\mathbf{x}}}{\mathrm{d} t} \mathbf{u}^{i} \nabla N^{i}(\xi, \eta)
$$

where $\mathrm{d} \hat{x} / \mathrm{d} t$ are the nodal velocities described earlier. The last term on the right hand side of Eq. (39) could be viewed as a correction term to account for nodal movement due to the deformation of the free boundary in an unsteady flow situation.

A special case arises for the time derivative of the free surface elevation $h$. In fixed isoparametric terms, the quantity $h$ can be interpreted as the isoparametric coordinate $\eta$, which is constant along the free boundary. Thus, the rate of change of $h$ in the isoparametric space is zero, since $\eta$ does not depend on time. It follows, that the local derivative of $h$ with respect to time becomes

$$
\frac{\partial h}{\partial t}=-\frac{\mathrm{d} \hat{\mathbf{x}}}{\mathrm{d} t} \cdot \nabla \eta
$$

in isoparametric coordinates and $\nabla \eta$ is normal to the free boundary.

The time derivatives of $\mathbf{u}$ are approximated by a difference scheme, as follows

$$
\dot{\mathbf{u}}^{n}=\frac{\theta}{\Delta t} \mathbf{u}^{n}+(1-\theta) \dot{\mathbf{u}}^{n-1}-\frac{\theta}{\Delta t} \mathbf{u}^{n-1}
$$

where superscript $n$ denotes that the variable is evaluated at time $t^{n}, \Delta t=t^{n}-t^{n-1}$, $\mathbf{u}$ represents the derivatives with respect to time and $\dot{\mathbf{u}}^{n-1}, \mathbf{u}^{n-1}$ are known from the solution of the previous time step. It is obvious now that when $\theta=1$, Eq. (41) reduces to a backward difference approximation and that when $\theta=2$, the scheme is identical to the trapezoidal rule, which is exclusively used in the present model, thus maintaining second-order accuracy with respect to the time increment of integration.

Each value of the indices appearing in Eqs. (31) to (35) represents the contribution of the non-linear equations to the element stiffness matrix. The local matrices are assembled to yield the global system of equations for the nodal unknowns, which can be represented as
$\mathbf{F}=\sum_{k=1}^{\mathrm{NE}} \mathbf{F}_{\text {domain }}^{\mathrm{e}}-\sum_{k=1}^{\mathrm{NB}} \mathbf{F}_{\text {boundary }}^{\mathrm{e}}$

in which NE is the total number of elements in the computational domain and NB is the total number of elements having a boundary segment on $\partial \Omega$.

The resulting system of algebraic equations is nonlinear, and an iterative procedure needs to be employed for its solution. Convergence of the iteration is not guaranteed unless the procedure is initiated within the radius of convergence, which is not in general known apriori. For steady flow problems, this is a very difficult problem (Schamber 1979), but in the present model, the process is much simpler. Due to the small time increments used in the computation, a good initial guess for the iterative solution is available from the last computational step. Newton's method converges in 3-5 iterations with the iteration tolerance set equal to $10^{-4}$ for the primitive variables. Defining the vector of the unknown expansion coefficients at the nodal points as

$\mathbf{R}=\left(\mathbf{U}^{\mathrm{T}}, \mathbf{v}^{\mathrm{T}}, \mathbf{P}^{\mathrm{T}}, \mathbf{h}^{\mathrm{T}}, \mathbf{k}^{\mathrm{T}}, \varepsilon^{\mathrm{T}}\right)$

the Newton iteration process finds the updated coefficient $\mathbf{R}^{n+1}$ from the last computed set $\mathbf{R}^{n}$ by solving the linear system of the equations

$J N^{n}\left(\mathbf{R}^{n+1}-\mathbf{R}^{n}\right)=-\mathbf{F}\left(\mathbf{R}^{n}\right)$

where $J N^{n}=(\partial \boldsymbol{F} / \partial \boldsymbol{R})^{n}$ are the elements of the Jacobian matrix. These have been presented in detail by Schamber (1979), Kistler and Scriven (1983) and Tsaras (1986).

\section{Simulation of the surge front}

The main difficulty in the mathematical modeling of the irrigation wave in the vertical plane is associated with the computation of the free surface. Initially the channel bed is dry and the wave is introduced at the upstream end of the field. A surge is immediately formed due to the interplay of intertial, frictional and gravitational forces. The basic mechanism for the propagation of the wave depends on the underlying assumptions for the dynamics of the flow. For shallow-water flow, a satisfactory model is obtained if the velocity near the front is assumed uniform (Whitham 1954). For vertically structured flow, the mechanism for propagation is more complicated. The model pro-

Fig. 1 Schematic of wave front movement

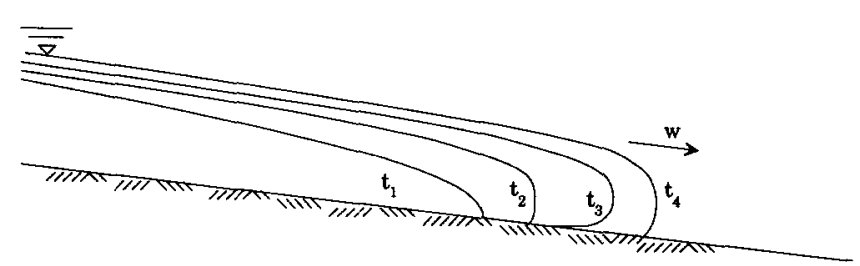


Fig. 2 a, b Finite element scheme for border flow with continuous free surface. a Finite element mesh. b Velocity vector field

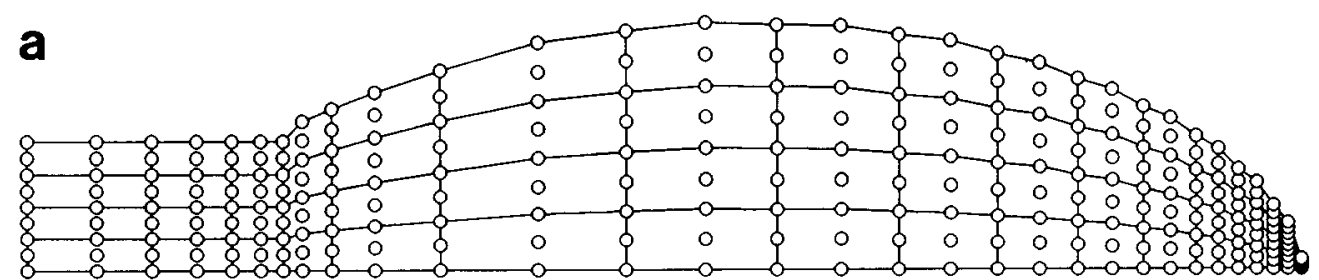

b
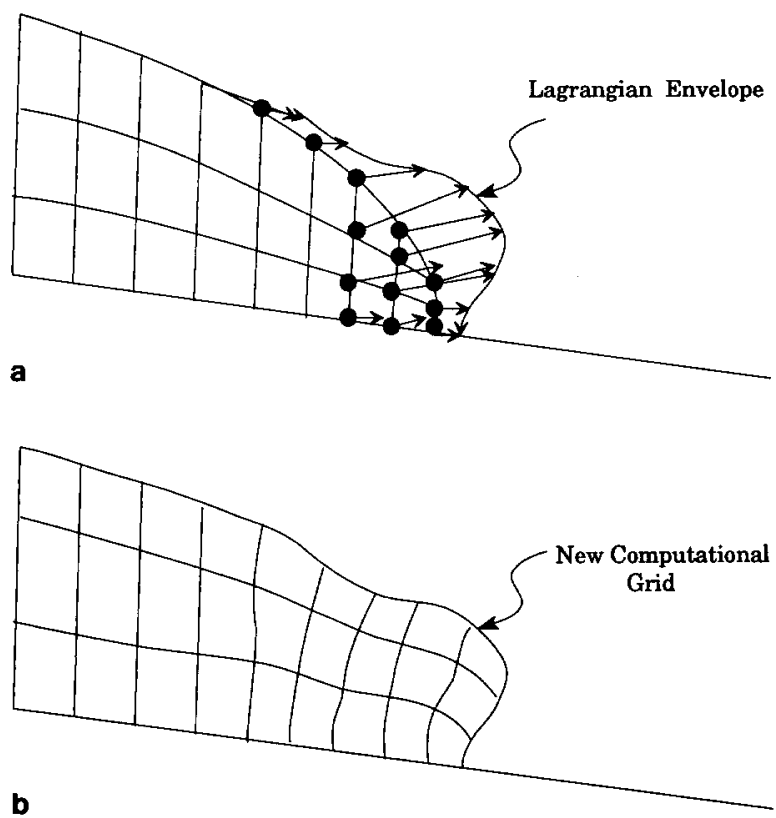

Fig. 3 a, b Computation of wave front movement. a Migration of frontal nodes. $\mathbf{b}$ Reshaped finite elements

posed in this paper suggests a rolling movement of water particles near the front resulting from the velocity profile in the vertical direction. Near the bed, fluid particles are retarded because of bed resistance. Streamlines are also deflected because of infiltration, especially near the wave front. Away from the bed, fluid particles move faster and then roll over the slow moving particles until they themselves become part of the bed zone where they are in turn resisted by bed friction. Figure 1 shows a schematic of this motion with the wave front undergoing a finite shape deformation. In reality this movement is continuous, so the front maintains an almost constant shape once the driving forces achieve equilibrium. The kinematic free-surface condition has been successfully used in problems associated with oscillatory free-surface flow in an open channel and in the configuration of surface shapes associated with laminar flows (Khesghi and Scriven 1981). For low values of the Reynolds number, the method proposed for steady flow by Tsaras (1986) has been successfully extended to unsteady flow. A uniform flow section is placed at the upstream end of the flow domain to facilitate the development of the deforming grid. The depth at the entrance is arbitrary, but taken equal to normal depth for convenience. A fully developed velocity profile is imposed at the first section. The grid is refined near the end of the fully developed flow and the top row of nodes is fixed, that is, a rigid-lid boundary condition is imposed on that segment of the flow. In the front segment, the free surface is given an arbitrary configuration and then adjusted in the course of the numerical solution. More nodes are concentrated near the wave tip, and nodal movement is restricted so that sections perpendicular to the bed are maintained. Figure $2 \mathrm{a}$ shows the finite element computational mesh associated with this technique. Figure $2 b$ shows the corresponding velocity field. Unfortunately, its use in turbulent flows has proved to be insufficient. The reasons are more of numerical than theoretical origin. Large deformations of the free surface near the wave front result in distorted shapes of the finite elements and eventually abortion of the computation. To avoid these problems, Tang (1991) used the Marker-and-Cell method proposed by Harlow and Welch (1965) and showed that in the numerical computation fluid particles penetrate the free surface from within the fluid domain, thus rendering the kinematic free surface condition invalid. There seems to be a continuous breaking of the wave front in turbulent flow, which, although maintaining approximately a fixed shape, cannot be tracked by postulating that fluid particles maintain their position on the free surface. Tang (1991) modified the Marker-and-Cell method to use irregular cells near the channel bed by use of finite element interpolation functions, which formed the basis for the method used in this paper. To avoid the difficulty with the boundary conditions of finite-difference methods and to reduce the number of markers required to locate the free surface, the present finite element model treats the nodal points near the front as markers, in a manner similar to the finite-difference tech- 
Fig. 4 Computed velocity profiles of rolling wave front
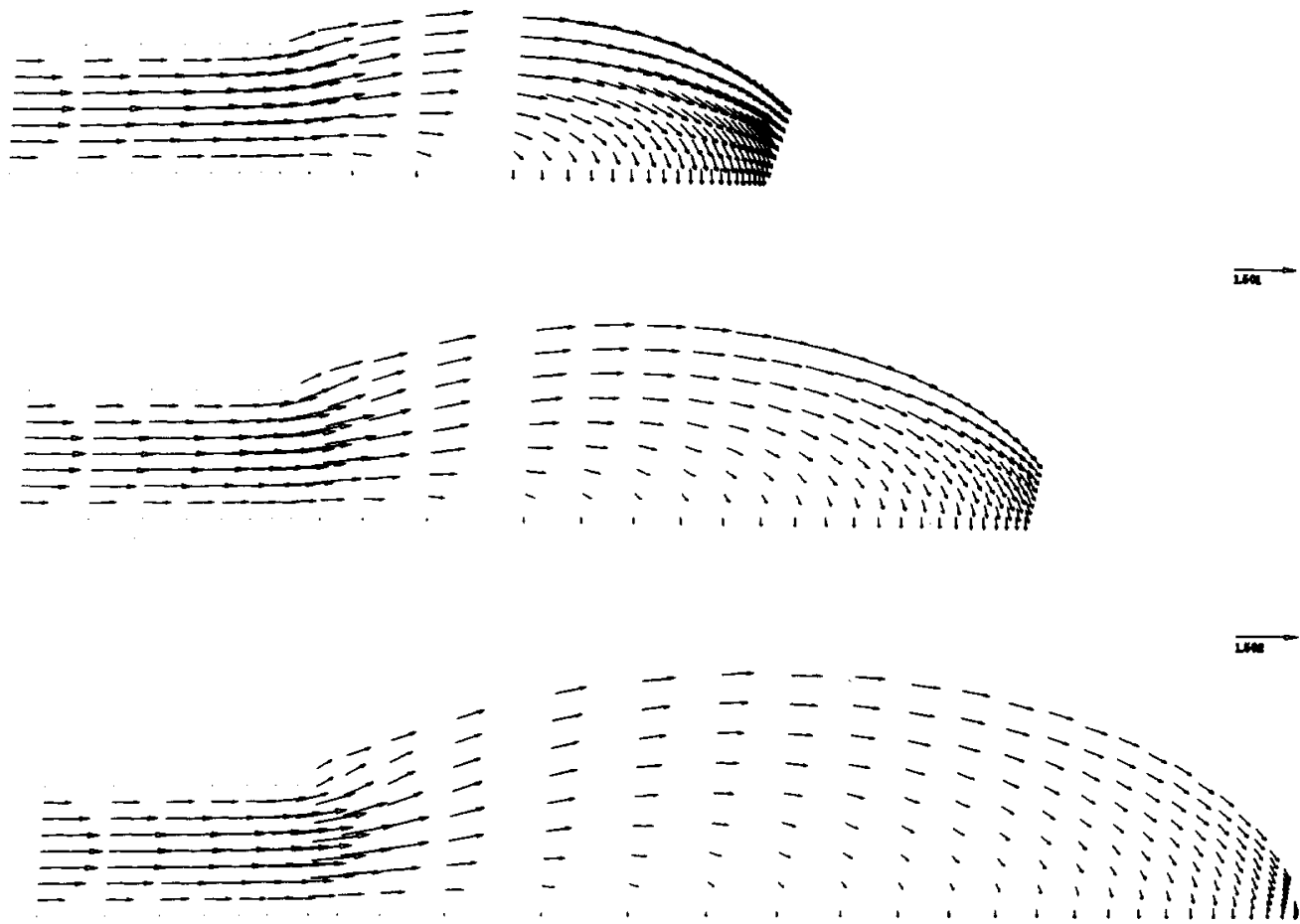

nique used by Miyata (1986) to account for wave breaking in coastal zones. Figure 3 shows the basic approach used in the computation. At the end of each time step, the nodal points near the wave front are moved with the fluid velocity computed by the finite element solution. As shown in Fig. 3 a, a Lagrangian envelope is thus traced around the new position of the nodes, and this is adopted as the new position of the wave front. The computational grid is reconstructed based on the boundary points and therefore the shape of the elements remains regular at all times, as shown in Fig. 3 b. Figure 4 shows the computed velocity fields resulting from the model, in which the rolling action of the wave front is obvious.

\section{Discussion of computational results}

The $k-\varepsilon$ model in the form employed in this paper has been verified for steady, uniform channel flow by Schamber (1979), and Tang (1991). Comparisons with experimental measurements (Alfrink and van Rijn 1983) indicate good agreement of computed and observed turbulent quantities. The applications of the present model concern surface irrigation waves, which differ only in the determination of the wave front position, as discussed above. For demonstration purposes, a well-known border irrigation experiment was analyzed with the following input data specifications (Katopodes and Strelkoff 1977). The inflow rate is $3.281 / \mathrm{s} / \mathrm{m}$, the bottom slope is 0.00101 , the Manning coefficient of roughness is 0.014 and Kostiakov's infiltration constants are $1.85 \mathrm{~cm} / \mathrm{min}$ and 0.24 , respectively. The only modification of input data for the Navier-Stokes model is the use of an equivalent roughness height, $k_{s}$, of $2.58 \mathrm{~mm}$ and an upper limit on the maximum infiltration rate, which is arbitrarily set equal to $50 \%$ of the maximum inflow velocity. Both the success of the iteration scheme and the rate of convergence were found to be strongly influenced by the very high values of infiltration near the wave front. Although the problem needs further investigation, the upper limit adopted in this paper was chosen to facilitate convergence. For the element sizes present in the computations, larger values of infiltration are encountered only at the tip node of the wave front. Thus the upper limit imposed results in an error of volume conservation, but not necessarily in the shape of the profile.

At the channel entrance, a fully developed velocity profile is specified, according to Eq (19). The shear velocity $u_{*}$ is set equal to an average value of $0.0473 \mathrm{~m} / \mathrm{s}$, computed from numerical tests in uniform channel flow. The water depth at the arbitrary inflow section is equal to $2 \mathrm{~cm}$ and von-Karman's constant $\kappa$ is equal to 0.4 . The boundary conditions for the turbulent quantities are based on the assumption that the normal derivatives of $\kappa$ and $\varepsilon$ vanish across the flow boundaries. In addition, the surface proximity function proposed by Gibson and Rodi (1989) is used to modify the vertical turbulent fluctuations near the free surface.

At the channel bed, the boundary conditions for $\mathrm{k}$ and $\varepsilon$ are specified based on Eqs. (17) and (18). At the free surface, normal derivatives of all turbulent variables are required to vanish, so the transport equations may conserve 
Fig. 5 Advance trajectories of wave front

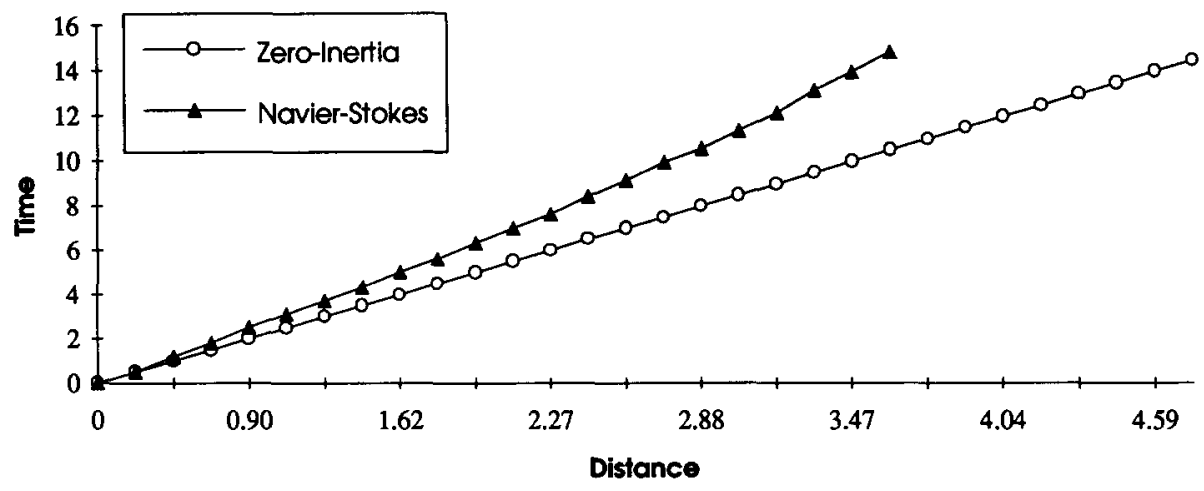

Fig. 6 Computed free-surface profiles

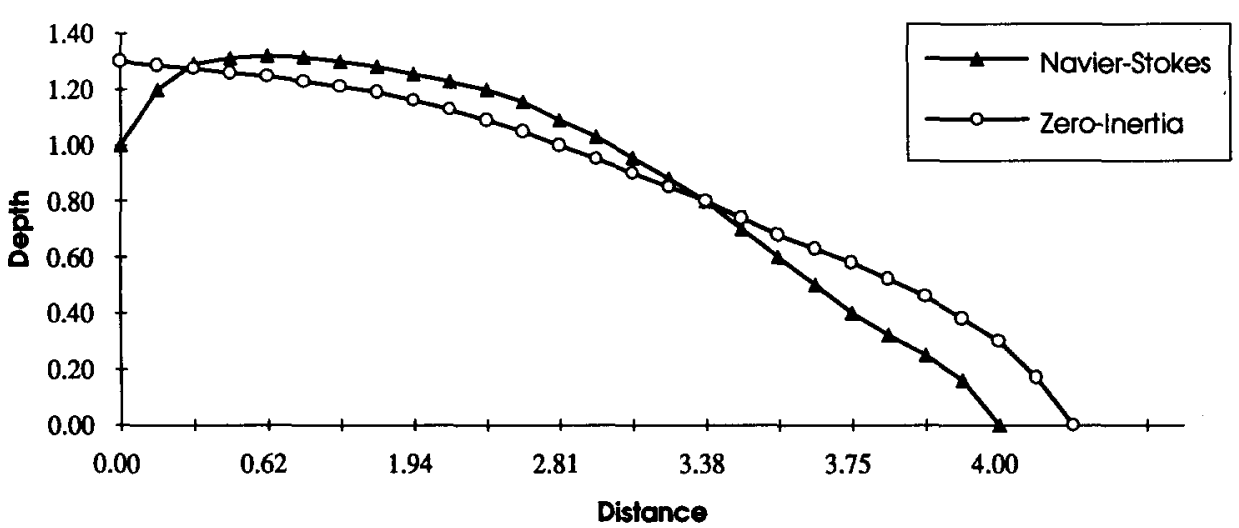

the kinetic energy and its rate of dissipation. The shear velocity at the bottom is initially given the same value as in the inflow section. Once, however, a tentative velocity profile is computed, the shear velocity is re-computed based on the actual near-bed velocity gradient. The process is repeated until convergence is obtained, which is achieved in five to six iterations during the first time step. Using previously computed values as a first guess results in significantly faster convergence during subsequent time steps.

The computations start by constructing a suitable but arbitrary initial condition for the problem. An initial wave form is developed by assuming that the front has propagated an arbitrary distance on a bed with a time-independent infiltration rate. The initial computational grid is assumed to correspond to an arrested wave front and is given a rectangular shape. Solution of the governing equations in steady form yields a tentative stationary position for the free surface, which satisfies continuity and all boundary conditions. The infiltration rate is corrected in the following time steps, thus reducing the infiltrating volume. The excess surface water then produces a transient front with a finite propagation velocity.

The rate of wave front advance was found to depend strongly on the infiltration capacity and equivalent roughness height. Numerous tests (Tang 1991) have shown that if the wave is allowed to reach a uniform outflow section, normal depth is eventually achieved. This uniquely determines the field characteristics based on standard resistance and infiltration formulas. The rate of advance, however, also depends on the numerical mechanism used to produce the rolling motion of the front by constructing the La- grangian envelope of moving nodal points. Comparison with shallow-water models indicate that the present scheme underestimates the rate of advance by $15-20 \%$. Figure 5 shows a comparison of the computed advance trajectory with that of the well-known Zero-Inertia model (Strelkoff and Katopodes 1977). The results are made dimensionless by a characteristic depth equal to $2 \mathrm{~cm}$, a length equal to $19.8 \mathrm{~m}$ and a time equal to $120.7 \mathrm{~s}$, based on normal depth considerations. The profiles shown in Fig. 6 are for dimensionless time $t=12$. The Navier-Stokes profile is pinned at $h=1$ at the upstream end, since the fully developed flow is assumed to be uniform. The Zero-Inertia model on the other hand, computes the upstream depth dynamically and has not yet reached normal conditions. The Navier-Stokes profile increases in depth a short distance downstream, but then falls short compared to ZeroInertia. The kinematic free-surface condition is a hyperbolic type equation and requires an upstream boundary condition, so there is little choice at the inflow boundary. The Navier-Stokes profile occupies also a somewhat smaller volume, but that is easily attributed to higher infiltration rates achieved near the wave front, since the tip node has a vertical velocity boundary condition, in contrast to Zero Inertia, which used a rigid body front, whose shape is not affected by infiltration.

Since the Zero-Inertia model agrees well with experimental measurements, the discrepancy in the two models is attributed to inaccuracies in the present model's propagation scheme. This can be improved by fine-tuning the rolling scheme of the front. In the present routine, for example, the front surface is forced to touch the channel bed 
Fig. 7 a, b Effects of increased wave speed on free-surface shape. a Standard front rolling action. b Accelerated front rolling action a

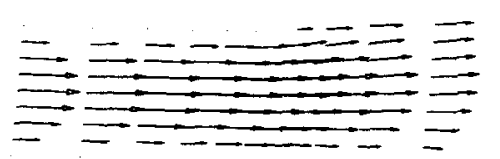

b

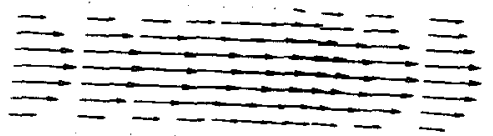

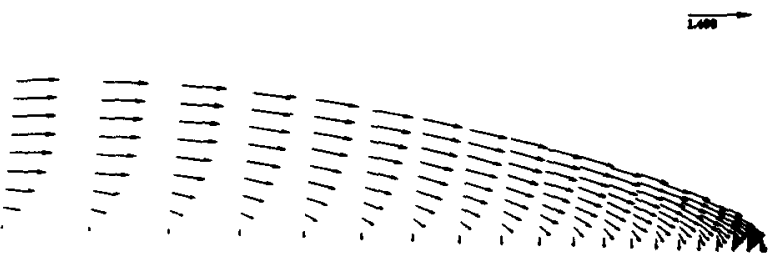

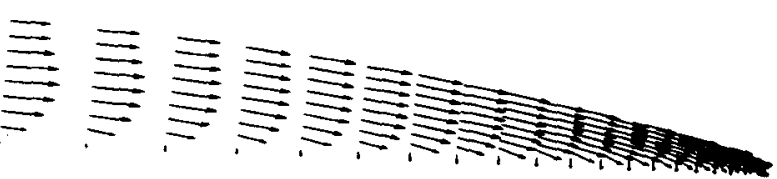

once the distance between the bed and the nearest node becomes smaller than a certain value. By increasing the numerical value of this arbitrary constant, the rolling motion is expedited resulting in a faster rate of advance. Unfortunately, this also alters the shape of the entire wave, as shown in Figs. $7 \mathrm{a}$ and $7 \mathrm{~b}$. The effect of the enhanced rolling action on the velocity field is to elongate the entire wave shape. Mass conservation obviously forces the faster waves to assume shallower depths that may not correspond to the true wave shape. This indicates the need for a more robust and perhaps physically correct approach for handling the advance process, although it may be difficult to exactly duplicate results of shallow-water models due to the significant differences in boundary conditions and parameters. Over small distances, the discrepancy between the two models is not great, so the procedure adopted in this paper is to terminate the two-dimensional computations a short distance away from the front. If the inflow rate at a section near the front is supplied by a shallow-water model at all times, the Navier-Stokes model can be used to compute the vertical structure of the front only. The technique gives excellent advance results for lengths corresponding to real borders and at the same time results in significant reduction in computational effort.

In summary, the present model presents a first attempt to model surface irrigation with a model based on the turbulent Navier-Stokes equations. In doing so, it provides information, previously unavailable from shallow-water models, that can be used for tracing the path of chemicals placed in irrigation water or introduced in the surface flow by suspension of contaminated soil particles. Several novel features have been introduced by combining the experience obtained from a steady flow finite element model and that from a transient marker-and-cell finite-difference method. The scheme introduced for the wave front advance on a dry bed is satisfactory, but requires further development. The present model is not suggested as a model to analyze or design border irrigation flow. In fact, it cannot compete with any of the state-of-the-art models available. Instead, it addresses a problem of greater dimension and importance, that of non-point source contamination from ir- rigation runoff. Since resistance, infiltration, turbulence and suspension of particles are intensified in the front region, the model is most effective when used in combination with a shallow-water model for the fully developed flow away from the front. There a suitable vertical velocity distribution may be used for vertical mixing purposes, since the pressure does not deviate from hydrostatic.

Acknowledgements The work presented in this paper has been supported by the Agricultural Research Service, U.S. Department of Agriculture, under cooperative agreement 59-5344-1-126. The writer would also like to acknowledge the contributions of his former students Y. Tsaras and J.-H. Tang, who developed the early versions of the Finite Element and Marker-and-Cell models.

\section{References}

Alfrink BJ, van Rijn LC (1983) Two-equation turbulence model for flow in trenches. ASCE J Hydr Engng 109:941-957

Chapman RS, Kuo CY (1985) Application of the two-equation $k-\varepsilon$ turbulence model to a two-dimensional, steady, free surface flow problem with separation. Inter J Numer Methods Fluids 5:257268

Finnie JI, Jeppson RW (1991) Solving turbulent flows using finite elements. ASCE J Hydr Engng 117: 1513-1530

Gibson MM, Rodi W (1989) Simulation of free surface effects on turbulence with a Reynolds stress model. J Hyd Res 27: 233-244

Harlow FH, Welch EJ (1965) Numerical calculation of time-dependent viscous incompressible flows of fluid with free surface. Physics Fluids 8: 2182-2194

Hood P, Taylor C (1974) Navier-stokes equations using mixed interpolation. Proceedings, International Symposium on Finite Element Method in Flow Problems, Swansea, Wales

Hughes TJR, Brooks A (1982) A theoretical framework for PetrovGalerkin methods with discontinuous weighting functions: Application to the streamline-upwind procedure. Finite Elements Fluids 4:47-63

Huh C, Scriven LE (1971) Hydrodynamic model of steady movement of a solid-liquid-air contact line. J Colloidal Interfacial Sci $35: 85$

Katopodes ND, Strelkoff T (1977) Hydrodynamics of border irrigation - complete model. ASCE J Irrig Drain Div 103:309-324

Khesghi FJ, Scriven LE (1981) Penalty finity element method for time dependent incompressible free surface film flow. In: Kawahara M (ed) Finite elements in fluid flow problems. Tokyo, Japan 
Kistler SF, Scriven LE (1983) Coating flow computations. In: Pearson JR, Richardson SM (eds) Computers in polymer processing. Applied Science Publishers Ltd, London

Leone JM, Gresho PM (1979) Finite element simulations of steady, two dimensional, viscous incompressible flow over a step. J Comput Phys 32:312-348

Lynch DR, Gray WG (1980) Finite element simulation of flow with deforming regions. J Comput Phys 36:2, Academic Press, New York

Miyata H (1986) Finite-difference simulation of breaking waves. J Comput Phys 65: 179-214

Ramaswamy B, Kawahara M (1987) Arbitrary lagrangian-eulerian finite element method for unsteady, convective, incompressible viscous free surface fluid flow. Int $J$ Numeric Methods Fluids 7: $1053-1075$

Rodi W (1988) Turbulence models and their application in hydraulics - a state-of-the-art review. International Association for $\mathrm{Hy}$ draulic Research, Delft, The Netherlands

Sakkas JG, Strelkoff TS (1974) Hydrodynamics of surface irrigation advance phase. J Irrig Drain Div 100:31-48
Schamber RD (1979) Finite element analysis of flow in a sedimentation basin. Ph.D Dissertation, Dept of Civil Engin, Univ of California, Davis

Schlichting H (1979) Boundary layer theory. Translated by Kestin J, Seventh edn, McGraw-Hill Co, New York

Strelkoff T, Katopodes ND (1977) Border irrigation hydraulics with zero inertia. ASCE J Irrig Drain Eng 103:325-342

Tang J-H (1991) Surge propagation on a porous bed. Thesis presented to the University of Michigan in partial fulfillment of the requirements of the degree of Ph.D

Tinney RE, Bassett DL (1961) Terminal shape of a shallow liquid front. ASCE J Hydraulic Eng 5: 117-133

Tsaras YG (1986) Finite element analysis of surge propagation on a porous bed. Thesis presented to the University of Michigan, in partial fulfillment of the requirements of the degree of Ph.D

Whitham GB (1954) The effects of hydraulic resistance in the dam break problem. Proc R Soc (London) Series A 227:399-407 